\title{
ÉTUDE DU DÉVELOPPEMENT DE CORDAGALMA CORDIFORMIS TOTTON, 1932, SIPHONOPHORE PHYSONECTE, AGALMIDAE
}

\author{
par \\ DANIELE CARRE \\ C.N.R.S., Station Zoologique, 06230-Villefranche-sur-Mer, France
}

\begin{abstract}
RESUME
L'étude morphologique et histologique des premiers stades larvaires du Siphonophore physonecte Cordagalma cordiformis montre que la segmentation aboutit à une périblastula qui se transforme en gastrula par délamination primaire. Au stade planula, une grande bractée larvaire apicale se différencie tandis que le pneumatophore se développe à partir de l'invagination précoce de quelques cellules ectodermiques.
\end{abstract}

\section{SUMMARY}

Histological studies of the first developmental stages of the physonect Siphonophore, Cordagalma cordiformis, shows that the segmentation leads to a periblastula changing into a gastrula by primary delamination. At the planula stage, a large apical bract appears and the pneumatophore develops from a precocious invagination of some ectodermal cells.

\section{INTRODUCTION}

Les grandes lignes du développement larvaire des Siphonophores physonectes étaient considérées comme connues depuis le mémoire de Metschnikoff, paru en 1874. Nous avons tenté de compléter ce travail portant sur la morphologie de quelques développements, en nous intéressant à d'autres espèces, et en utilisant la méthode de l'histogénèse comparée jusque là totalement négligée. Nous nous sommes particulièrement intéressés à la segmentation des oeufs, ainsi qu'aux modalités de la gastrulation, phénomènes sur lesquels les données étaient fragmentaires et peu explicites. Nous avons également suivi la différenciation des principaux organes afin de pouvoir discuter de l'existence d'homologies entre eux (Carré, D., 1967, 1969, 1971). C'est dans le cadre de ce travail que se situe l'étude morphologique et histologique du développement de Cordagalma cordiformis Totton, 1932, Siphonophore Physonecte Agalmidae.

\section{MATERIEL ET METHODES}

Cordagalma cordiformis est un petit siphonophore (30 à 40 centimètres de longueur totale) présentant une vaste répartition géographique mais dont la morphologie n'a pu être précisée que récemment (Carré, C., 1968). En effet ses colonies sont extrêmement fragiles et ne peuvent être obtenues en bon état qu'en les prélevant directement dans la mer à l'aide d'un récipient.

Les méthodes d'élevage des adultes et des larves ainsi que les techniques histologiques sont celles décrites dans nos précédentes publications.

\section{L'OEUF ET LA SEGMENTATION}

Les gonophores femelles de Cordagalma cordiformis sont de petits médusoïdes subsphériques de $500 \mu$ s'insérant chacun sur le stolon par un pédoncule propre, et dont le manubrium renferme un oeuf unique de $300 \mu$ de diamètre.

Comme tous les oufs de siphonophores que nous avons observés, l'oeuf de Cordagalma cordiformis, à maturité, est constitué de deux parties nettement distinctes: une région périphérique, ou ectoplasme, formée de cytoplasme finement granuleux qui contient le noyau, et une région interne vacuolaire, l'endoplasme, où sont localisées les réserves vitellines. Après l'émission des globules polaires au pôle distal de l'oeuf, le gonophore se détache du stolon et tombe sur le fond du cristallisoir; quelques heures plus tard, la paroi du manubrium se déchire et l'oeuf est libéré.

La fécondation se produit aussitôt après la ponte et la segmentation débute une dizaine de minutes plus tard avec l'apparition du premier plan de clivage au pôle de maturation; ce plan partage l'oeuf en deux blastomères égaux qui s'écartent l'un de l'autre en ne restant réunis que par un pédoncule 


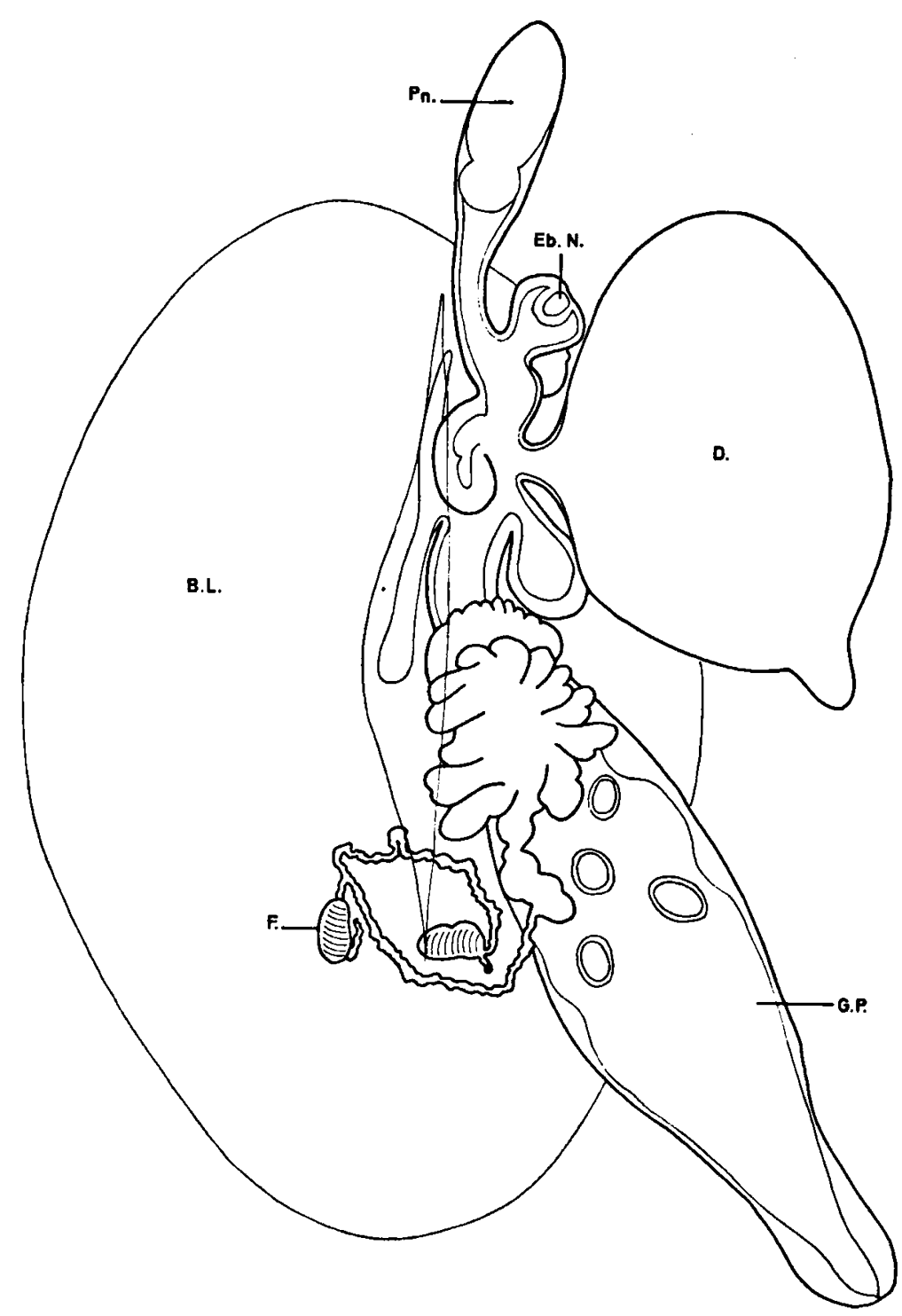

Fig. 1. Siphonula de Cordagalma cordiformis âgée de 15 jours. Abréviations: voir planche II.

cytoplasmique. Puis ces deux blastomères se rapprochent et s'accolent tandis que s'ébauche le second plan de segmentation en position équatoriale. Le troisième clivage suit un plan perpendiculaire aux précédents et partage l'oeuf en huit blastomères semblables formant deux quartettes superposés. La quatrième segmentation s'effectue suivant deux plans méridiens et perpendiculaires entre eux qui délimitent seize blastomères. Au-delà de ce stade, il devient difficile de repérer l'orientation relative des plans de clivage; cependant nous avons pu observer, cinq heures après la fécondation, le passage par une série de divisions radiaires et synchrones, à une larve formée de trente-deux blastomères. A ce stade, la différence de plasme notée dans la structure de l'oeuf est maintenue; chaque blastomère présente une calotte externe d'ectoplasme contenant le noyau, et une région interne formée uniquement d'endoplasme anuclée. Cette larve, encore dépourvue de ciliature reste une à deux heures sur le fond du cristallisoir avant le début d'une nouvelle série de mitoses plus ou moins synchrones mais s'effectuant toutes suivant des plans radiaires. Lors de ces nouvelles segmentations, seu- 

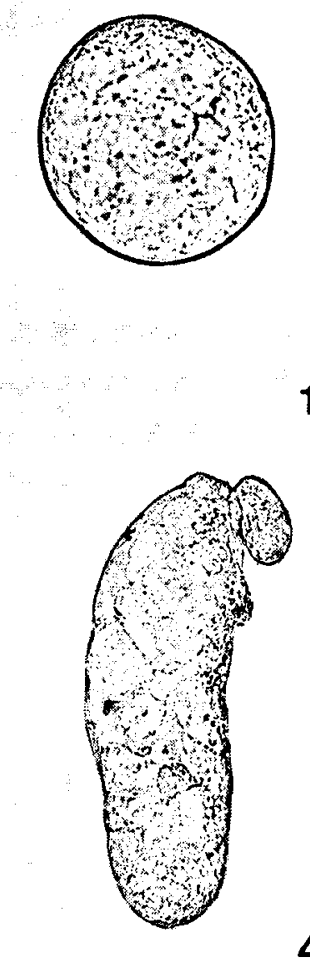

1

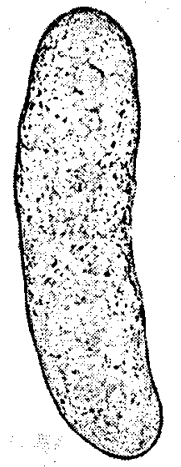

2
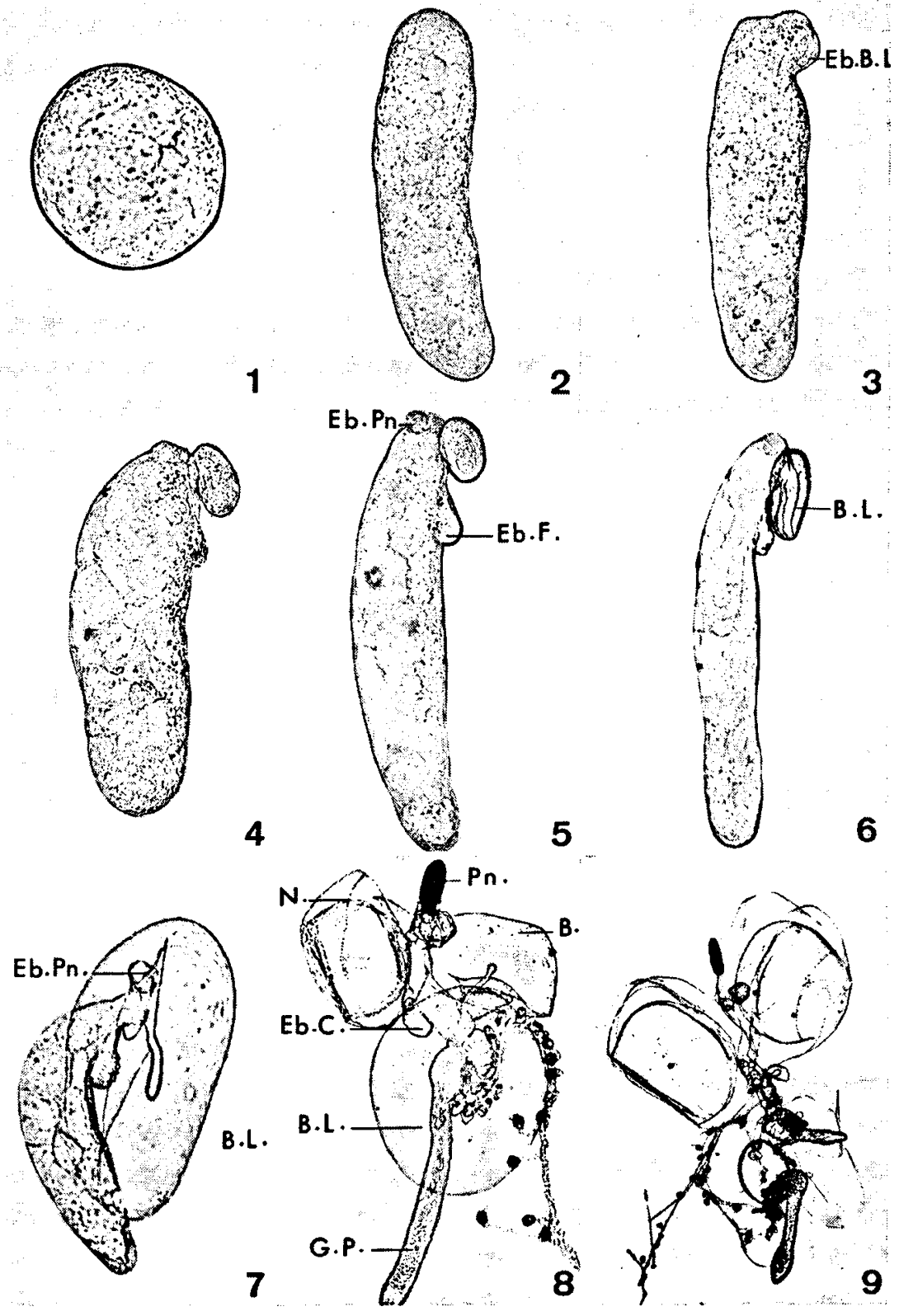

5

6

Planche I.

1, morula; 2, planula âgée d'1 jour; 3, ébauche de la bractée larvaire chez une planula âgée de 2 jours; 4, planula âgée de 2 jours $1 / 2 ; 5$, ébauche du pneumatophore

chez une planula âgée de 3 iours; 6, jeune siphonula; 7 , siphonula âgée de 6 jours; 8 , post larve âgée de 3 semaines; 9 , jeune colonie âgée d'1 mois.

Abréviations: voir planche II.

le la région ectoplasmique des blastomères se divise en même temps que les noyaux tandis que l'endoplasme n'est plus concerné par les mitoses. Ceci aboutit à la formation d'une enveloppe constituée de soixante-quatre cellules plates entourant la masse du vitellus (PI. I, 1; Pl. II, 1). 


\section{LA GASTRULATION}

Au-delà du stade à soixante quatre blastomères le synchronisme des mitoses disparait. La plupart des cellules continuent à se segmenter suivant des plans radiaires mais certaines se divisent tangentiellement à la surface de la larve, isolant de l'assise superficielle, qui formera l'ectoderme, des noyaux entourés d'un peu de cytoplasme finement granuleux qui sont les ébauches du feuillet endodermique. Ces noyaux endodermiques restent contre la face interne de l'ectoderme, sauf quelques-uns qui migrent au sein de l'endoplasme.

Ce phénomène de gastrulation par délamination primaire typique dure environ une heure et s'achève en donnant une planula longue, mince et ciliée (Pl. I, 2; PI. II, 2).

\section{LA PLANULA}

Le développement de la planula de Cordagalma cordiformis montre les stades déjà décrits dans nos précédents travaux sur le développement des Siphonophores physonectes et nous nous bornerons à signaler quelques particularités.

Deux jours après la ponte, la planula présente un épaississement apico-ventral au niveau duquel l'ectoderme et l'endoderme s'évaginent pour donner l'ébauche de la bractée larvaire qui est le premier organe à se différencier (PI. I, 3; Pl. II, 3). Puis, tandis que cette ébauche s'extériorise et se pédiculise, on observe, en position apico-dorsale l'invagination de quelques cellules ectodermiques qui pénètrent à l'intérieur du corps embryonnaire où elles forment l'ébauche du flotteur. Les premiers stades du développement de cette ébauche sont identiques à ceux des pneumatophores dont nous avons étudié la formation (Pl. II, 4, $5,6)$.

$\mathrm{Au}$ cours de sa différenciation le pneumatophore devient apical et fait saillie à l'extérieur de la larve qui est devenue une jeune siphonula.

\section{LA SIPHONULA}

\section{Transformation de la jeune siphonula}

La bractée larvaire dont l'ébauche est apparue au-dessus du pneumatophore, grandit rapidement pour former, chez la larve âgée de six jours, un bouclier ovale de $0,8 \mathrm{~mm}$ de long, qui enveloppe l'ébauche du pneumatophore et une partie du corps embryonnaire (Pl. I, 4, 5, 6, 7). Cette bractée est formée par une épaisse couche de mésoglée limitée par l'ectoderme; elle possède un canal bractéal rectiligne. Au cours de son développement les réserves vitellines régressent à l'intérieur du corps embryonnaire dont les parois se différencient en un gastrozoïde primaire. En coupe histologique, on observe dans l'endoderme du gastrozoïde des cellules spumeuses hypostomiales et des cellules sphéruleuses hypostomiales et gastriques. A la base du gastrozoïde, l'ébauche du filament pêcheur se développe, elle s'allonge en une formation ecto-endodermique très contractile sur laquelle se différencient les ébauches des tentilles (Pl. I, 7, 8).

A ce stade, la cavité définitive remplie de gaz apparaît au sein du tissu aérifère du pneumatophore qui s'éloigne de la bractée larvaire.

\section{La jeune colonie.}

La région de la larve comprise entre le pneumatophore et la zone d'insertion de la bractée larvaire s'étire en un axe creux, ébauche du stolon, sur lequel bourgeonne le premier nectophore qui présente tous les caractères spécifiques des cloches natatoires des colonies adultes (PI. I, 8). Simultanément, à l'opposé de l'insertion de la bractée larvaire sur le stolon, se développe le dactylozoïde primaire (Fig. 1). Puis apparaissent, sous la zone de bourgeonnement des nectophores, les éléments de la deuxième cormidie: gastrozoïde, dactylozoïde, filament pêcheur, et bractée en tronc de pyramide identique à celles des colonies adultes (PI. I, 8).

Nous avons pu poursuivre les élevages pendant plus d'un mois et obtenir de petites colonies pourvues de deux grands nectophores et de plusieurs cormidies (P1. I, 9). Nous avons également pu observer la chute du bouclier primaire ce qui confirme son caractère larvaire.

\section{CONCLUSIONS}

Nos observations sur les premiers stades du développement de Cordagalma cordiformis sont en accord avec celles faites lors de l'étude des développements de Nanomia bijuga et d'Halistemma rubrum. Nous avons noté à chaque fois une 


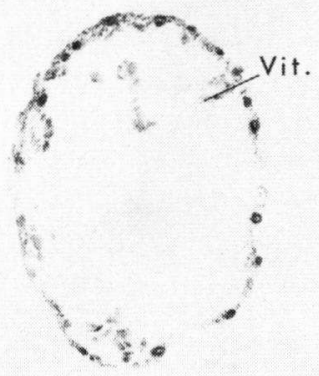

1

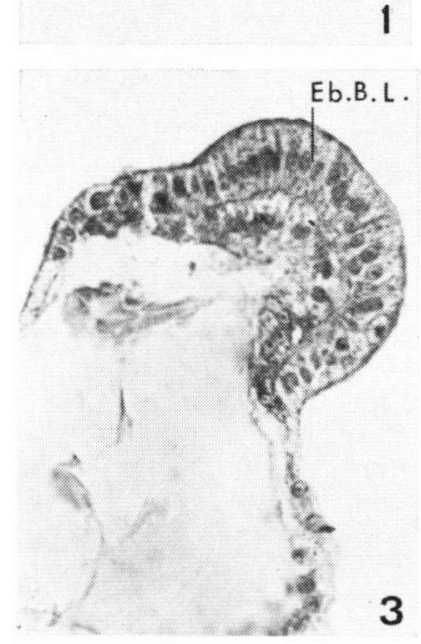

3
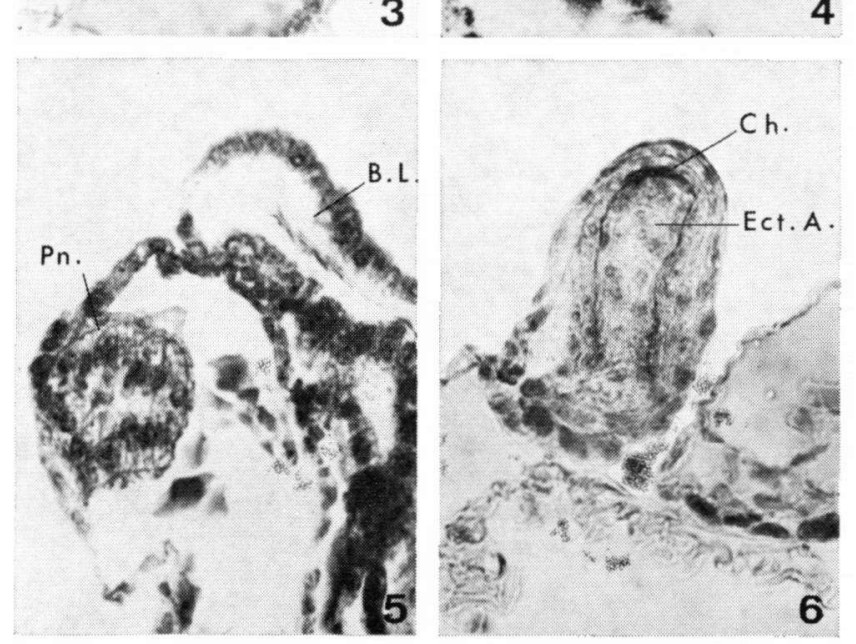

Abréviations:

B Bractée définitive

B.L. Bractée larvaire

Ch. Chitine

D. Dactylozoïde

Eb.B.L. Ebauche de la bractée larvaire

Eb.C. Ebauche de la deuxième cormidie

Eb.F. Ebauche du filament pêcheur

Eb.N. Ebauche de nectophore

Eb.Pn. Ebauche du pneumatophore

Ect.A. Ectoderme aérifère

F. Filament pêcheur

G.P. Gastrozoïde primaire

N. Nectophore

N.end. Noyau endodermique

Pn. Pneumatophore

Vit. Vitellus
Planche II.

1, périblastula; 2 , gastrula; 3 , ébauche de la bractée larvaire; 4 , invagination du pneumatophore; 5 , région anté- rieure d'une jeune siphonula; 6 , coupe longitudinale dans un pneumatophore avant l'apparition de la cavité définitive. 
segmentation totale et égale puis partielle et superficielle conduisant à la formation d'une périblastula.

L'existence d'un bouclier larvaire identique à celui déjà signalé chez Agalma elegans (Metschnikoff, 1874) permet de rapprocher le genre Cordagalma du genre Agalma, et l'écarte, parmi les
Agalmidae, des genres Halistemma et Nanomia dont les larves ne possèdent pas de bractée larvaire (Metschnikoff, 1874; Carré, D., 1969, 1971).

Enfin, la connaissance de ce développement facilite la détermination des jeunes stades de Cordagalma cordiformis rencontrés dans les pêches planctoniques.

\section{INDEX BIBLIOGRAPHIQUE}

Carre, C., 1968. Description d'un Siphonophote Agalmidae, Cordagalma cordiformis Totton, 1932. Beaufortia, 16 (212) : 79-86.

Carré, D., 1967. Etude du développement larvaire de deux Siphonophores Lensia conoïdea et Forskalia edwardsi. Cah. Biol. mar., 8 : 233-251.

—, 1969. Etude histologique du développement de Nanomia bijuga (Chiaje, 1841), Siphonophore Physonecte Agalmidae. Cah. Biol. mar., 10 : 325-341.
-, 1971. Etude du développement d'Halistemma rubrum (Vogt, 1952) Siphonophore Physonecte Agalmidae. Cah. Biol. mar., 12 : 77-93.

Metschnikoff, E., 1874. Studien über die Entwicklung der Medusen und Siphonophoren. Zeit. wiss. Zool., $24: 15-83$.

Totron, A. K., 1954. Siphonophora of the Indian Ocean together with systematic and biological notes on related species from other oceans. Discovery Rep., 27 : $1-161$. 